\title{
Predictions of the electro-mechanical response of conductive CNT-polymer composites
}

\author{
M.A.S. Matos, V.L. Tagarielli*, P.M. Baiz-Villafranca and S.T. Pinho \\ Department of Aeronautics, Imperial College London, SW7 2AZ London, UK
}

\begin{abstract}
We present finite element simulations to predict the conductivity, elastic response and strainsensing capability of conductive composites comprising a polymeric matrix and carbon nanotubes. Realistic representative volume elements (RVE) of the microstructure are generated and both constituents are modelled as linear elastic solids, with resistivity independent of strain; the electrical contact between nanotubes is represented by a new element which accounts for quantum tunnelling effects and captures the sensitivity of conductivity to separation. Monte Carlo simulations are conducted and the sensitivity of the predictions to RVE size is explored. Predictions of modulus and conductivity are found in good agreement with published results. The strain-sensing capability of the material is explored for multiaxial strain states.
\end{abstract}

Keywords: strain-sensing (A); carbon nanotube (B); polymeric material (B); finite elements (C)

Submitted to Journal of the Mechanics and Physics of Solids, November 2017

*Corresponding author. E-mail v.tagarielli@imperial.ac.uk 


\section{Introduction}

The applications of carbon nanotubes (CNT) have recently received growing interest due to their good combination of mechanical, electrical, and thermal properties (Coleman et al.). Consisting of cylindrical arrangements of one (single-wall, SWNT) or multiple (multi-wall, MWNT) graphite sheets, CNTs have stiffness in the TPa range (Treacy et al.) and electrical conductivities between $10^{3}$ and $10^{7} \mathrm{~S} / \mathrm{m}$ (Ebbesen et al.). Due to small dimensions, very high aspect ratios and low mass density, the excellent properties of these nanostructures can be exploited to their fullest if dispersed into lightweight matrices as engineering polymers (Spitalsky et al.), allowing development of multifunctional materials for a wide range of applications (Baughman et al.). Small concentrations of CNTs (as low as $0.0025 \mathrm{wt} \%$ (Sandler et al., 2003)) can render polymers conductive, by forming percolating networks (Stauffer and Aharony). Depending on the polymer and production process, a filler fraction of $1 \mathrm{wt} \%$ is usually able to place the composite conductivity in the order of $1 \mathrm{~S} / \mathrm{m}$ (Bauhofer and Kovacs, 2009).

When subject to deformation, these conductive pathways are distorted, resulting in a change of the electrical properties of the composite. By measuring the change in bulk resistance, the composite can operate as a self-sensing material that is multidirectional (Obitayo and Liu) and that possesses superior sensitivity to that of traditional resistance strain gauges (Pham et al., 2008).

Carbon nanotubes undergo structural and band gap changes when subject to mechanical strain (Tombler et al.) and therefore their deformation can be detected by changes in their Raman spectra (Frogley et al.). Alternatively, when CNTs are embedded in a polymeric matrix, the resulting bulk electrical conductivity of the composite is sensitive to macroscopic strain (Dharap et al.; Zhang et al.), enabling real-time self-sensing. It is widely accepted (Alamusi et al.) that the piezoresistivity of CNT-polymer composites is linked to three major mechanisms: (i) the inherent piezoresistivity of CNTs (Park et al.), (ii) the deformation of the conductive paths that they form within the polymer and (iii) the change of inter-filler distances, directly influencing the tunnelling phenomena responsible for conductivity between adjacent nanotubes (Di Ventra; Kilbride et al., 2002).

Several modelling approaches have been proposed to predict the electro-mechanical response of CNT-polymer composites (Alamusi et al.), including semi-empirical and numerical models. For example, (Pham et al.) proposed a simple model relating applied strain to a decrease in effective 
volume fraction and a consequent decrease in conductivity (Stauffer and Aharony); (Park et al.) proposed a similar model which accounts for the effects of an increased average tunnelling distance between the CNTs at high strain; (Wichmann et al.) modelled a resistor network of parallel paths, including a sensitivity of tunnelling conductivity to strain; (Kuronuma et al.) used a similar approach but distinguished two types of contacts: overlapping and plane contacts. Some authors proposed micromechanics approaches limited to configurations of up to three nanotubes with

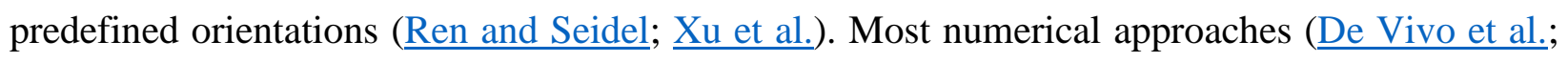
Gong and Zhu; Gong et al.; Hu et al.; Rahman and Servati) consider a 3D random network of resistors, with tunnelling resistors between neighbouring CNTs, and a fibre reorientation model (Taya et al.) to capture the effects of strain. More recently, (Panozzo et al.) proposed an analytical expression based on an equivalent network of parallel paths where CNTs are connected in series; the sensitivity to strain was computed based on an estimate of the average variation of the tunnelling separation distance, from inputs such as the undeformed electrical conductivity and some morphology parameters. Most authors considered the CNTs as rigid, while others modelled them as deformable, in accordance to continuum mechanics; (Grabowski et al.) recently proposed a multiscale approach where the elastic properties of CNTs are simulated with molecular dynamics.

A survey of the literature shows that the main current challenges include ability: to model a realistic microstructure; to include curved CNTs; to explore the effects of matrix conductivity; and to obtain predictions which do not rely on several fitting parameters, that need calibrating against measurements. In this study we address these challenges: we model microstructures of arbitrary complexity, including a network of curved CNTs; we perform sequentially-coupled, physicallybased detailed analyses of both the mechanical and electrical response of the composites; we introduce the effects of quantum tunnelling at the junctions between CNTs, developing a new quantum tunnelling element based on the constitutive relation from Simmons; we explore the sensitivity of the predictions to RVE size; and we study the phenomenon of self-sensing in different load cases. 


\section{Construction of the RVEs}

The FE analyses simulate the electro-mechanical response of representative volume elements comprising a homogeneous matrix and a network of CNTs of arbitrary complexity. The RVEs consist of cubic volume elements of side length $L_{\mathrm{RVE}}$; the CNTs are represented by penalized basis splines ( $\mathrm{p}$-splines) and have diameter $D_{\mathrm{CNT}}$. A target volume fraction $V_{\mathrm{f}}$ and the statistical distributions of nanotube dimensions and orientation are prescribed; the $i$-th nanotube is generated from a sequence of straight segments (up to the desired length $L_{\mathrm{CNT}_{i}}$ ) originating at a random location within the RVE, of coordinates generated by uniformly distributed pseudo-random numbers between 0 and 1 . The first segment is assigned random azimuthal $\left(\alpha_{0}\right)$ and latitudinal $\left(\phi_{0}\right)$ angles; subsequent $j$-th segments are randomly generated within the three-dimensional cone $\theta_{j} \leq \theta_{\max }$, where $\theta_{j}$ is the angle formed by the $j$-th segment and the preceding $((j-1)$-th) segment, as illustrated in Fig. 1. The angle $\theta_{\max }$ is used to control the waviness of the nanotubes.

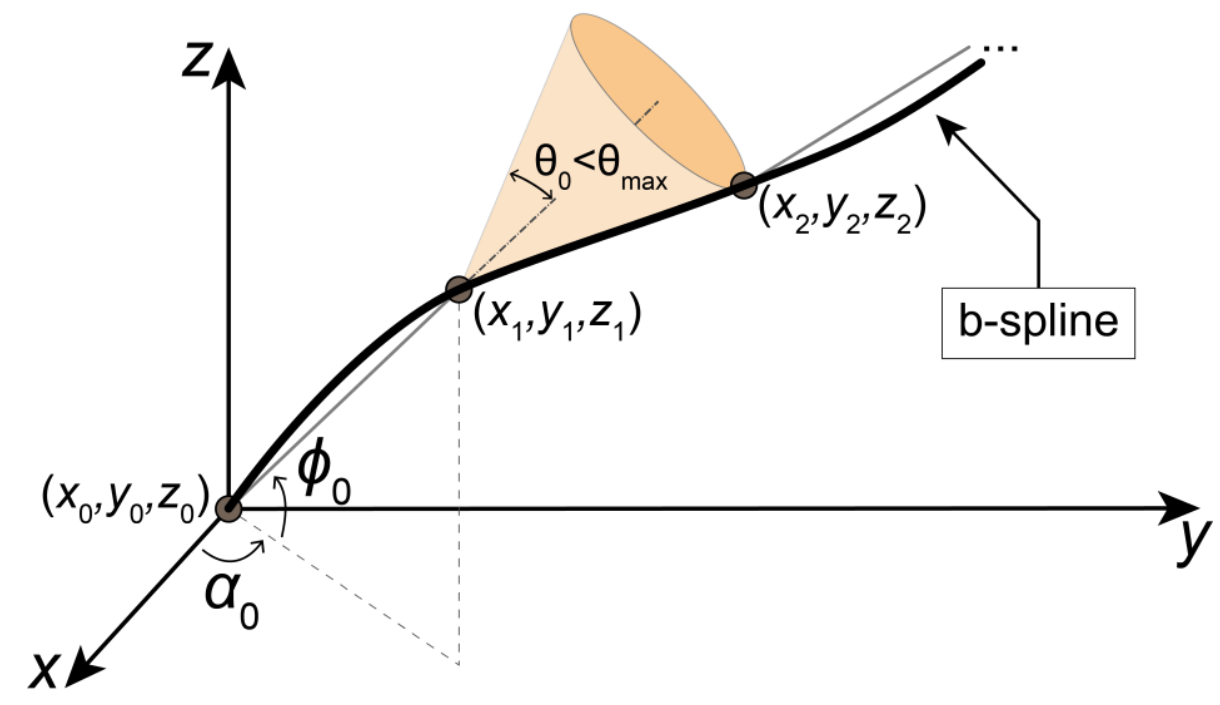

Fig. 1. Illustration of the generation of a CNT.

The segments are then replaced by p-splines sharing the intermediate endpoints; geometry periodicity is enforced by repositioning the sections that override the domain boundaries. Since the nanotubes are impenetrable, a search for intersections between CNTs is performed; when intersections are detected, the corresponding splines are locally perturbed so that the minimum 
distance that separates two nanotubes is never smaller than the Van der Waals equilibrium distance of $d_{\mathrm{VdW}}=0.34 \mathrm{~nm}$ (Girifalco et al.; Yoon et al.). This guarantees that there is no force between the CNTs in the initial configuration and assumes that the CNTs are not separated by a polymer molecule. Further mechanical interaction between pairs of CNTs is not modelled, although it has been shown that local contact may affect the mechanical and electrical interaction between pairs of CNTs (Mirzaeifar et al.). As it will be shown below, in this study we model CNTs as one-dimensional beams; accounting for local deformation effects, while keeping the simplicity of the beam abstraction, would require additional parameters whose determination is not clear, as discussed by (Gong and Zhu, 2014).

An example of a generated RVE is displayed in Fig. 2: Fig. 2(a) shows the initial distribution of p-splines, corresponding to different CNTs; in Fig. 2(b), geometric periodicity has been enforced.

a)

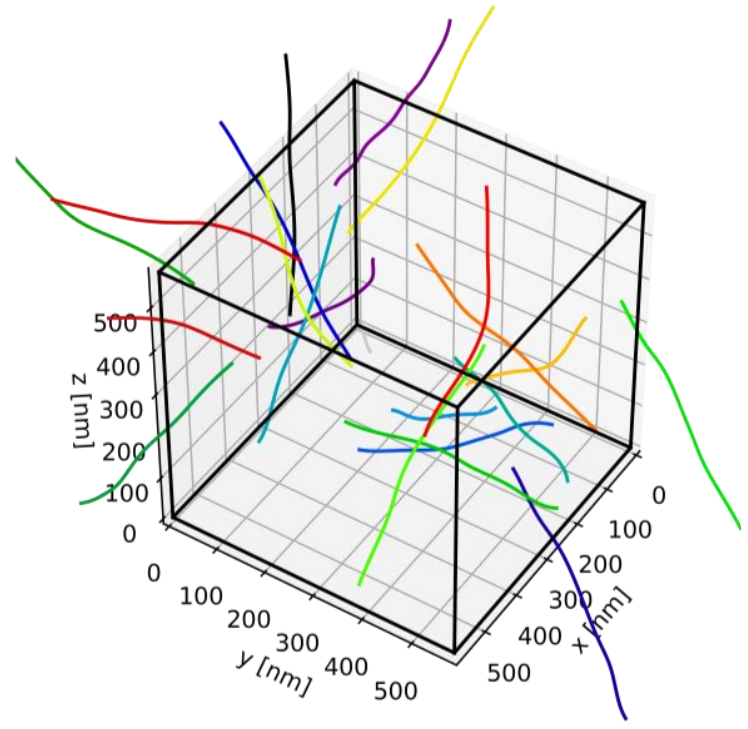

b)

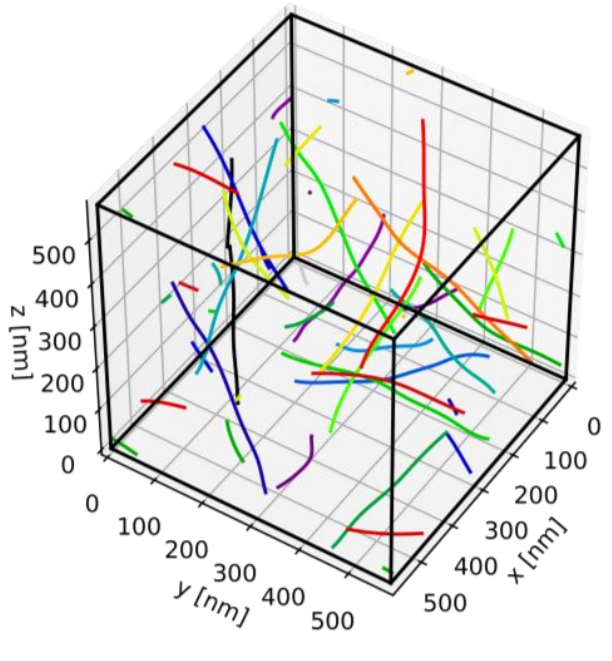

Fig. 2. Example of an RVE geometry: a) before and b) after enforcing geometry periodicity.

To locate intersections, an approach similar to spatial filtering (Gonzalez and Woods) is used, as illustrated (in 2D) in Fig. 3. The domain is divided into equally-sized cubic raster cells with side length $d_{\text {min }}=D_{\mathrm{CNT}}+d_{\mathrm{VdW}}$; the splines are seeded by points separated by a distance $D_{\mathrm{CNT}} / 10$. Each point can be linked to the raster cell it belongs to, allowing easy identification of near neighbours. Intersection is detected when the minimum distance between points of different CNTs is smaller than the grid size $d_{\text {min }}$. A similar procedure is used to detect electrical junctions; these are defined as locations where the distance between the centre-lines of two adjacent CNTs is such 
that tunnelling conduction may occur; this is found by searching for all pairs of adjacent CNTs

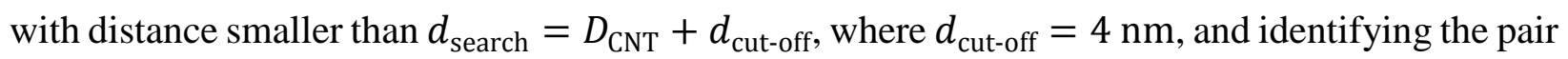
of points with minimum distance. Nodes are placed corresponding to the pairs of points defining each tunnelling junction, for subsequent meshing.

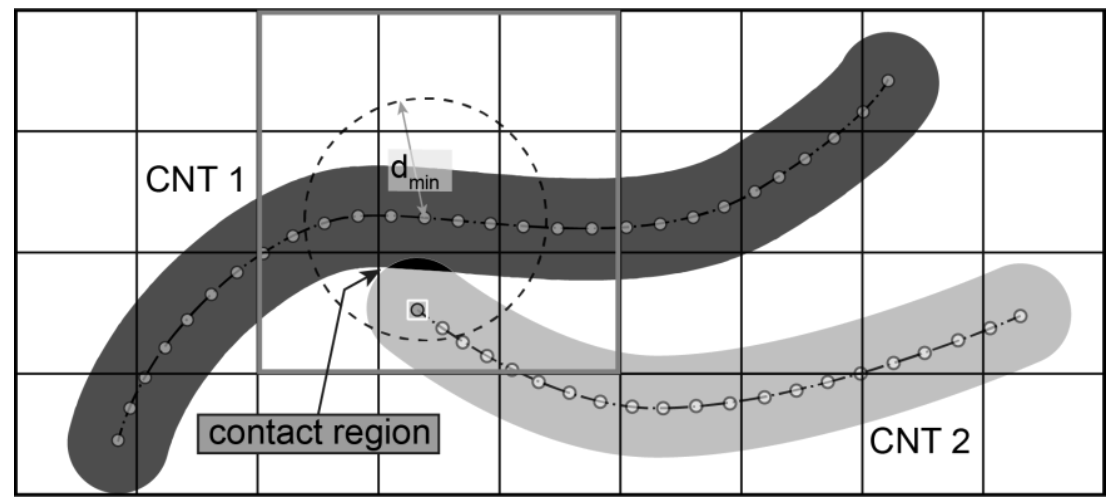

Fig. 3. Illustration in $2 \mathrm{D}$ of the procedure to identify contact points.

While this approach is focused on a uniform dispersion, the initial CNT configuration could be, in principle, obtained from simulation methods such as Dissipative Particle Dynamics (Wescott et al.), however at a much higher computational cost.

\section{Homogenization}

The homogenized response of the macroscopic structure can be estimated based on the analysis of the RVEs after imposing periodic boundary conditions (PBCs). Below, we provide details of the homogenization schemes used for simulations of the mechanical and electrical response.

\subsection{Mechanical homogenization}

The constitutive equation for a liner elastic material can be written as

$$
\sigma_{i j}(\mathbf{x})=C_{i j k l}(\mathbf{x}) \varepsilon_{k l}(\mathbf{x})
$$

where $\mathbf{X}$ represents the position vector and $\sigma_{i j}, C_{i j k l}$ and $\varepsilon_{k l}$ the stress, stiffness, and strain tensors. The latter is related to the displacement field $\mathbf{u}$ by 


$$
\varepsilon_{k l}=\frac{1}{2}\left(u_{k, l}+u_{l, k}\right)
$$

The averaged stress $\bar{\sigma}_{i j}$ and strain $\bar{\varepsilon}_{k l}$ tensors can be defined as spatial averages of the local distributions over the representative volume $V$

$$
\bar{\sigma}_{i j}=\frac{1}{V} \int_{V} \sigma_{i j} \mathrm{~d} V
$$

and

$$
\bar{\varepsilon}_{k l}=\frac{1}{V} \int_{V} \varepsilon_{k l} \mathrm{~d} V
$$

Similarly, we can rewrite equation (1) in terms of averaged quantities

$$
\bar{\sigma}_{i j}=\bar{C}_{i j k l} \bar{\varepsilon}_{k l}
$$

Equation (5) indicates that the homogenized properties $\bar{C}_{i j k l}$ can be computed based on the RVE deformation from the averaged (or homogenized) stress and strain tensors.

Since the macroscopic material can be represented by an infinite array of repeated RVEs, deformation of the RVE should be spatially periodic. This is enforced by prescribing PBCs along its boundaries. Following the approach described by ( $\underline{\text { Suquet}), ~ t h e ~ s t r a i n ~ t e n s o r ~ c a n ~ b e ~ e x p a n d e d ~}$ into average $\left(\bar{\varepsilon}_{k l}\right)$ and fluctuation $\left(\varepsilon_{k l}^{*}\right)$ terms, the latter having vanishing spatial average over the RVE volume

$$
\varepsilon_{k l}=\bar{\varepsilon}_{k l}+\varepsilon_{k l}^{*}
$$

Integrating equation (6), assuming an irrotational displacement field and making use of equation (2), the displacement at any given point is

$$
u_{k}=\bar{\varepsilon}_{k l} x_{l}+u_{k}^{*}
$$


where $x_{l}$ is the component of the position vector along $l$; $u_{k}^{*}$ can be shown to be periodic (Suquet) and therefore has the same value at homologous points of opposite boundaries. Considering two opposite surface boundaries of the RVE with positive $(l+)$ and negative $(l-)$ normals along the $X_{l}$ axis, the respective displacements can be related as

$$
u_{k}^{l+}-u_{k}^{l-}=\bar{\varepsilon}_{k m}\left(x_{m}^{l+}-x_{m}^{l-}\right)=\bar{\varepsilon}_{k l} L_{\mathrm{RVE}}
$$

where the quantity in parentheses is exactly the RVE side length if $l=m$ and zero otherwise. Equation (8) is enforced through constraint equations that relate the degrees of freedom (DOF) of each pair of homologous nodes on opposite boundaries to the 6 DOFs of a master node, corresponding to the 6 components of the homogenized strain tensor $\bar{\varepsilon}_{k l}$.

\subsection{Electrical homogenization}

The electrical flow of current $\mathbf{J}$ is described by Ohm's law and its constitutive relation can be written as:

$$
J_{i}(\mathbf{x})=\kappa_{i j}(\mathbf{x}) E_{j}(\mathbf{x})=-\kappa_{i j}(\mathbf{x}) \frac{\partial V}{\partial x_{j}}(\mathbf{x})
$$

with $\kappa_{i j}$ being the electrical conductivity tensor, $E$ and $V$ the electric field and potential. Following the same procedure as for the mechanical case, one can define average quantities of the current density and electric field, analogous to equations (3) and (4). Equation (9) can therefore be written in terms of homogenized values

$$
\bar{J}_{i}=\bar{\kappa}_{i j} \bar{E}_{j}=-\bar{\kappa}_{i j} \overline{\left(\frac{\partial V}{\partial x_{J}}\right)}
$$

and the electrical potential within a periodic RVE is

$$
V=\bar{E}_{k} x_{k}+V^{*}
$$


where $V^{*}$ is a periodic component of the electrical potential, having the same value at homologous points of opposite boundaries. Repeating the procedure described above, the periodic boundary conditions for the electrical potential can be written as

$$
V^{j+}-V^{j-}=\bar{E}_{j} L_{\mathrm{RVE}}
$$

Equation (12) is enforced at homologous boundary nodes via constraint equations relating the electric potential of the boundary nodes to the 3 components of the average electric field $\overline{\mathbf{E}}$. We note that this condition also implies that the total current flowing through opposite faces of the RVEs are equal and opposite.

\section{Finite Element simulations}

Two types of simulations are conducted, exploring the mechanical and electrical response of the RVE, respectively. The two types of simulations are then sequentially coupled in order to determine the strain-sensing response of the composite. In all cases, we perform Monte Carlo analyses: we consider multiple realizations (20 realisations unless otherwise stated) of the RVEs and repeat the corresponding simulations; then, we analyse the average and spread of the outputs. Details of the simulation techniques are presented below.

\subsection{Mechanical model}

The CNTs are represented as equivalent continuum fibres (Thostenson and Chou) and modelled as beams with a hollow cylindrical cross-section, using the B31 elements of Abaqus Standard (Simulia). The cross-section has the same diameter of the nanotubes and the thickness of each nanotube wall is set to $0.34 \mathrm{~nm}$ (Odegard et al.), corresponding to the interlayer spacing of graphene. This value has been the target of different studies, which reported values ranging from 0.0617 to $0.69 \mathrm{~nm}$ (Huang et al.) and dependent on the load case (Odegard et al.). We stress here that in this study CNTs are modelled as a structure rather than a material, specifically as a one-dimensional beam element. Axial and bending stiffness of CNTs are obtained from calculations at lower scale (Guo and Zhang; Wu et al.); in our FE simulations however, we input the geometry of a hollow circular cross-section, which in conjunction with the material's modulus, determines the effective axial and bending stiffnesses in the simulations. The choice of the wall 
thickness is therefore dependent on the choice of Young modulus. In this study the choice of a thickness of $0.34 \mathrm{~nm}$ was made to allow a direct comparison with the results from (Yuan and Lu); the material response is taken as isotropic and linear elastic.

The polymeric matrix is discretized into a regular mesh with 8-noded full integration brick elements (C3D8 in Abaqus). The relevant mechanical properties are listed in Table I. The nanotubes are assumed to be perfectly bonded to the matrix and modelled as embedded elements.

Mechanical PBCs are prescribed only at the boundary nodes of the matrix phase, since the nanotubes are constrained to this phase. Since the beams representing the CNTs also have rotational DOFs which are not constrained by the embedment, rotations of CNT intersecting the RVE boundaries are enforced to be equal at homologous nodes. Six load cases are imposed: 3 for pure uniaxial strain and 3 for pure shear deformation in 3 mutually perpendicular planes. The 6 components of the homogenized strain tensor are prescribed in turn and, for each, the corresponding homogenized stress tensor is determined from the reaction forces on appropriate auxiliary nodes. This allows assembly of the full stiffness matrix and calculations of the homogenized elastic engineering constants. For the case of isotropic RVEs, presented in the following, these reduce to the homogenized elastic modulus $\bar{E}_{\text {comp }}$ and Poisson's ratio $\bar{v}_{\text {comp }}$.

\subsection{Electrical model}

Since CNTs are good conductors, their dispersion within an insulating matrix will result in a conductive material if they form a conductive percolating network. Due to the large ratio between the conductivities of CNTs and matrix, there is a concentration threshold above which the composite is said to be conductive - the critical volume fraction $V_{\mathrm{f}_{\mathrm{c}}}$. The macroscopic conductivity will therefore depend on the intrinsic conductivity of the constituents, the paths they form and the resistance of the tunnelling junctions. While the conductivity of the polymer matrix is often neglected, due to its low value and the increased modelling complexity, the present methodology allows for its inclusion without added effort.

As in the mechanical model, the matrix is meshed by a regular tessellation; the nanotubes, modelled as one-dimensional conductors, are embedded within the matrix. This is done by linking the (voltage) degrees of freedom of the embedded CNT to the corresponding degrees of freedom of the matrix elements, via linear shape functions. The electrical analysis is performed using 
Abaqus steady-state heat-transfer analysis ( $\underline{\text { Simulia) }}$ since the constitutive equations for electrical

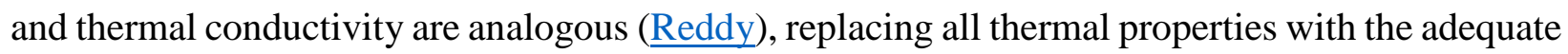
electrical equivalents. The matrix is discretized with DC3D8 8-noded brick elements and the CNTs are modelled by DC1D2 2-noded link heat transfer elements.

Electric PBCs are applied by enforcing a uniaxial homogenised electric field (or potential difference) along each of the 3 Cartesian directions, in three separate load steps. This allows constructing the full homogenised electrical conductivity matrix $\bar{\kappa}_{i j}$.

The resistance of all tunnelling junctions is modelled via a user element, described in detail in the next Section.

\subsubsection{Quantum tunnelling element}

Several authors have found experimental evidence (Kilbride et al.) that the conductivity between adjacent CNTs can be described by fluctuation-induced tunnelling electron transport (Di Ventra). The conductivity across two nanotubes is dependent on the probability of their electrons to penetrate and overcome the electric potential barrier between them and is here calculated using Simmons's generalized formula (Simmons). The tunnel current density $J$ across adjacent CNTs separated by a thin insulating layer of permittivity $\epsilon$ can be expressed as a function of the separation $s$ between them, as

$$
\begin{gathered}
J=J_{0}\left\{\bar{\varphi} \mathrm{e}^{-A \sqrt{\bar{\varphi}}}-(\bar{\varphi}+e V) \mathrm{e}^{-A \sqrt{\overline{\bar{\varphi}+e V}}}\right\} \\
J_{0}=\frac{e}{2 \pi h(\Delta s)^{2}} \text { and } A=\frac{4 \pi \Delta s}{h} \sqrt{2 m_{e}}
\end{gathered}
$$

where $e$ and $m_{e}$ represent the charge and mass of the electron, respectively, and $h$ is the Planck's constant. $\bar{\varphi}$ is the mean value of the potential barrier, which has two roots at positions $s_{1}$ and $s_{2}$, with $\Delta s=s_{2}-s_{1}$. For a potential barrier of magnitude $\varphi_{0}$ these can be approximated (with image force effects included) by (Simmons)

$$
\bar{\varphi}=\varphi_{0}-\left(\frac{e V}{2 s}\right)\left(s_{1}+s_{2}\right)-\left[\frac{1.15 \omega s}{s_{2}-s_{1}}\right] \ln \frac{s_{2}\left(s-s_{1}\right)}{s_{1}\left(s-s_{2}\right)}
$$




$$
\begin{gathered}
s_{1}=\frac{1.2 \omega s}{\varphi_{0}} \text { and } s_{2}=\left\{\begin{aligned}
& s_{1}+s {\left[1-\frac{9.2 \omega}{3 \varphi_{0}+4 \omega-2 e V}\right], e V<\varphi_{0} } \\
&\left(\varphi_{0}-5.6 \omega\right)\left(\frac{s}{e V}\right), e V \geq \varphi_{0}
\end{aligned}\right. \\
\omega=\frac{e^{2} \ln 2}{8 \pi \epsilon S}
\end{gathered}
$$

We take the potential barrier $\varphi_{0}$ equal to the CNT work function (the energy necessary to extract one electron from the CNT). Simmons's formula is valid in one-dimensional scenarios, such as in a parallel-plates capacitor; here, we describe the tunnelling junction between CNTs as an equivalent parallel-plate capacitor of area $D_{\mathrm{CNT}}^{2}$ and separation $s$ for any angle between crossing CNTs. This approximation neglects the effects of the circular shape of the CNT cross-section and of the angle between neighbouring CNTs. The choice is supported by the fact that (Nigro and Grimaldi) concluded that the effect of relative angle in resistive tunnelling processes is marginal for randomly dispersed CNTs, for all volume fractions. The assumption of a parallel-plate capacitor was found adequate in the case of the contact resistance measured for perpendicular SWNTs (Fuhrer et al.) at a separation of $s=0.34 \mathrm{~nm}$, coinciding with the van der Waals equilibrium spacing between adjacent graphene sheets.

The separation distance is related to the coordinates of the two contact nodes $P_{1}$ and $P_{2}$ as follows

$$
s=\max \left\{s_{\min }, \sqrt{\sum_{i=1}^{3}\left(P_{1_{i}}-P_{2_{i}}\right)^{2}}-D_{\mathrm{CNT}}\right\}
$$

Since compression between two nanotubes can decrease their minimum separation to $0.25 \mathrm{~nm}$, as reported by (Yoon et al. $), s_{\min }=0.25 \mathrm{~nm}$ is used throughout this study.

The resulting element is illustrated in Fig. 4, having 2 nodes and 1 DOF per node corresponding to the nodal electrical potential. Three constitutive parameters are assigned to each tunnelling element: potential barrier, relative permittivity and nanotube diameter; these allow relating the current $I^{\mathrm{N}}$ (nodal flux) to the electric potential $V^{\mathrm{N}}$ (nodal DOF) via the formulation presented above, linearized around the electric potential difference predicted by a parallel plate capacitor $V=e s /\left(\epsilon D_{\mathrm{CNT}}^{2}\right)$. The respective Jacobian (stiffness) matrix is computed as $K_{i j}=-d I^{\mathrm{N} i} / d V^{\mathrm{N} j}$, 
where $\mathrm{N} i$ refers to the $\mathrm{i}$-th node of the element, and the corresponding right-hand-side vector (or residual nodal flux) as $F_{i}=-I^{N i}$. This is integrated into a Fortran UEL subroutine in Abaqus (Simulia).

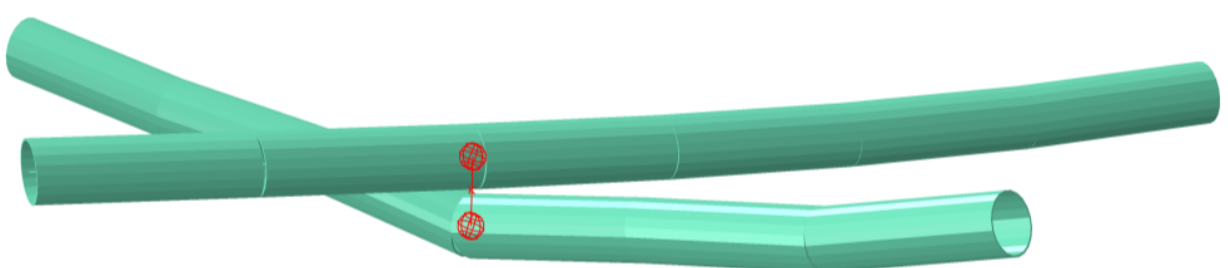

Fig. 4. Example of the user element applied at the contact point between two nanotubes.

\subsection{Strain-sensing}

As discussed by (Alamusi et al.) and mentioned in the introduction, the piezoresistivity of CNTreinforced polymers is attributed to the change of tunnelling junction separations and to the inherent CNT piezoresistivity, with the latter contribution often neglected. While the piezoresistivity of SWNT can vary from negative to positive values depending on chirality, the piezoresistivity of a MWNT is expected to be low. In addition, the high stiffness of CNTs and their isotropic distribution lead to a very small influence of CNT piezoresistivity to the overall change of the composite resistance, estimated to be less than 5\% (Oliva-Aviles et al.). Thus, the current approach focuses only on the first, dominant mechanism.

To model the piezoresistive response of the RVEs, the mechanical and electrical models are coupled sequentially. The mechanical model is employed to determine, for each of the $k$ tunnelling elements, the sensitivity $\left(S_{k}^{i j}\right)$ of the tunnelling separation $s_{k}$ to each of the imposed homogenized strain component $\bar{\varepsilon}_{i j}, S_{k}^{i j}=\partial s_{k} / \partial \bar{\varepsilon}_{i j}$ (dependent on the load case). This sensitivity is included in the electrical model as a property of each tunnelling element: the element is modified to have 8 DOFs: the electric potential at its two nodes and the 6 macroscopic strain components, defined as global DOFs. A schematic illustration of this is presented in Fig 5.

The same periodic boundary conditions described for the electrical analysis are applied; simulations of the strain-sensing response are conducted in two steps: (1) a constant electric field is applied to the RVE; (2) selected homogenized strain component $\bar{\varepsilon}_{i j}$ are prescribed under the 
constant electric field. By measuring the change in current flow in the direction of the applied electric field, resistance versus strain histories can be obtained.

We note that fully-coupled simulations can also be conducted on electro-mechanical models; however, a fully coupled approach is computationally less efficient than the proposed sequentially coupled technique; the two approaches yield identical outputs for small strains.

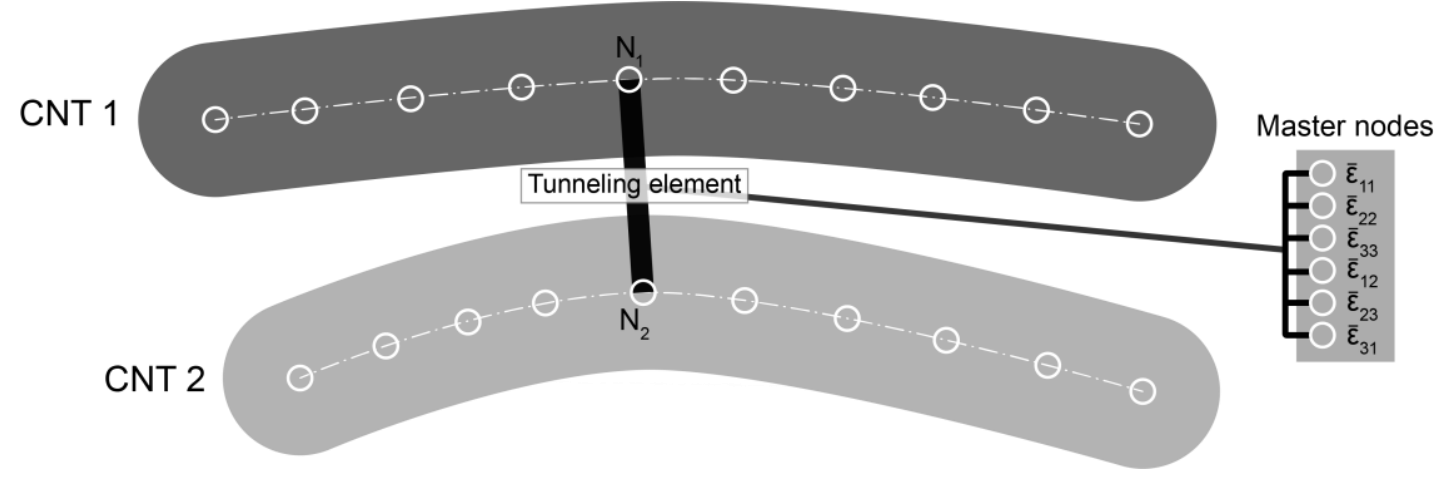

Fig 5. Schematic illustration of the user-defined tunnelling element.

\section{$5 \quad$ Results and discussion}

The proposed methodology is now applied to predict the response of different composites comprising single-wall and multi-wall nanotubes (SWNT and MWNT, respectively) dispersed in polymeric matrices; the predictions are compared to published results for validation of the proposed technique.

\subsection{Mechanical properties}

To validate the mechanical model, we use as a benchmark the results of a study by (Yuan and $\underline{\mathrm{Lu}}$ ), considering a random distribution of SWNTs in a polypropylene (PP) matrix; these authors considered the effects of nanotube waviness (Herasati and Zhang) on the macroscopic elastic modulus. Relevant dimensions and elastic properties are provided in Table I. A volume fraction of $V_{\mathrm{f}}=1.0 \%$ is considered. 
To guarantee a correct representation of the microstructure, an appropriate RVE size must be determined. To do so, the dependence of the homogenized modulus $\bar{E}_{\text {comp }}$ and Poisson's ratio $\bar{v}_{\text {comp }}$ (averaged in the three Cartesian directions) is explored, as shown in Fig. 6 for 20 realizations, number obtained after a convergence study.

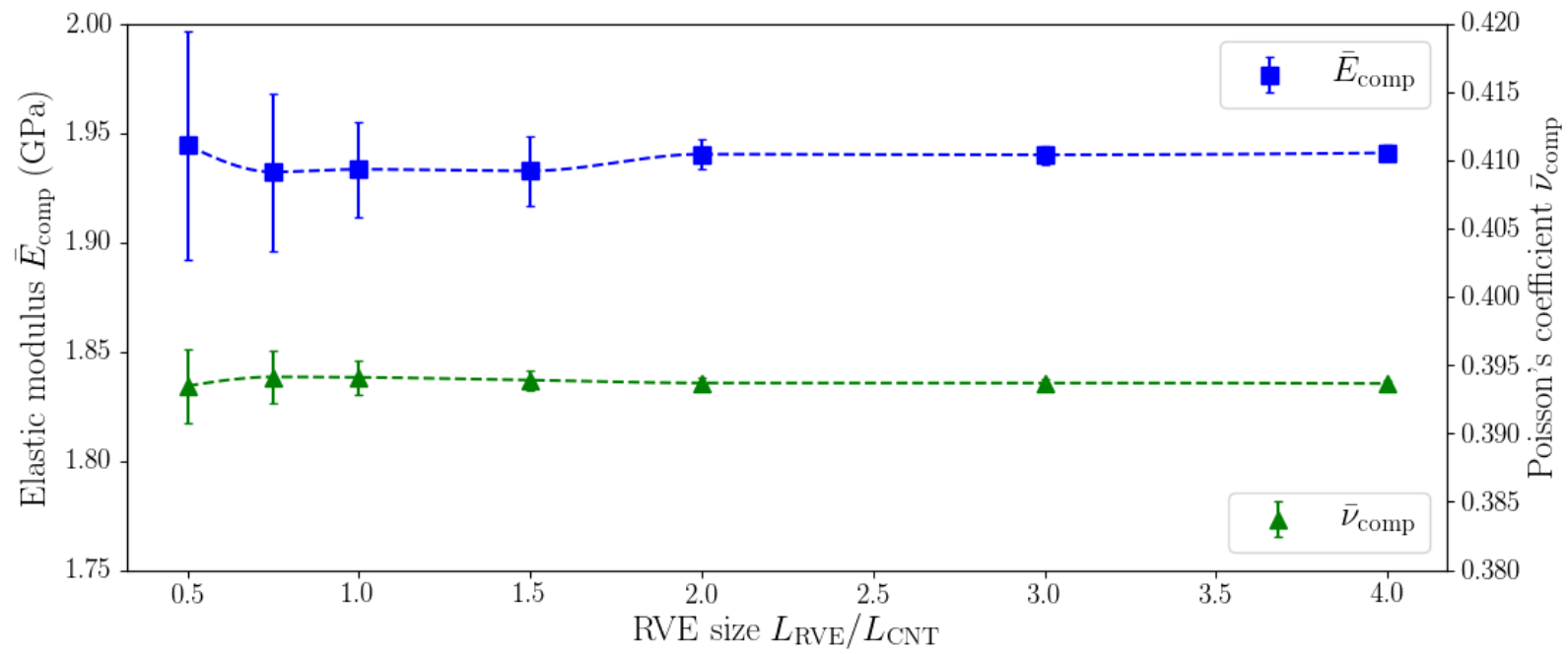

Fig. 6. Dependence of the homogenized elastic properties on the RVE size. The bars represent standard deviation.

The predicted elastic properties are insensitive to RVE size for $L_{\mathrm{RVE}} \geq 2.0 \times L_{\mathrm{CNT}}$; for $L_{\mathrm{RVE}}=2.0 \times L_{\mathrm{CNT}}$ the standard deviation is less than $0.5 \%$ of the mean. A mesh convergence study was also carried out for this RVE size, leading to FE models with approximately 1.1 million DOFs. The effect of waviness was explored by keeping the total length of the nanotubes constant and varying the maximum angle $\theta_{\max }$ between consecutive segments from $0^{\circ}$ to $25^{\circ}$; the corresponding effective elastic modulus is plotted in Fig. 7 against the average waviness ratio $\lambda=L_{\mathrm{CNT}} / L_{\mathrm{eff}}$, where $L_{\text {eff }}$ is the distance between the start and end points.

For straight CNTs $\left(\lambda=1\right.$, or $\left.\theta_{\max }=0^{\circ}\right)$, an average modulus of $\bar{E}_{\text {comp }}=1.941 \mathrm{GPa}$ is obtained, whereas (Yuan and Lu) report a value of $1.938 \mathrm{GPa}$, for the case of CNTs modelled as threedimensional solids (as opposed to beams). The sensitivity to waviness predicted by the current approach is also in line with the predictions presented in (Yuan and Lu) and shown in Fig. 7. The small offset between the two sets of predictions is at least partially due to the fact that results by (Yuan and Lu) consider a distribution of CNT lengths (however this is not clearly stated in their 
paper), while our set of predictions consider CNTs of length equal to the average used by (Yuan and $\mathrm{Lu})$.

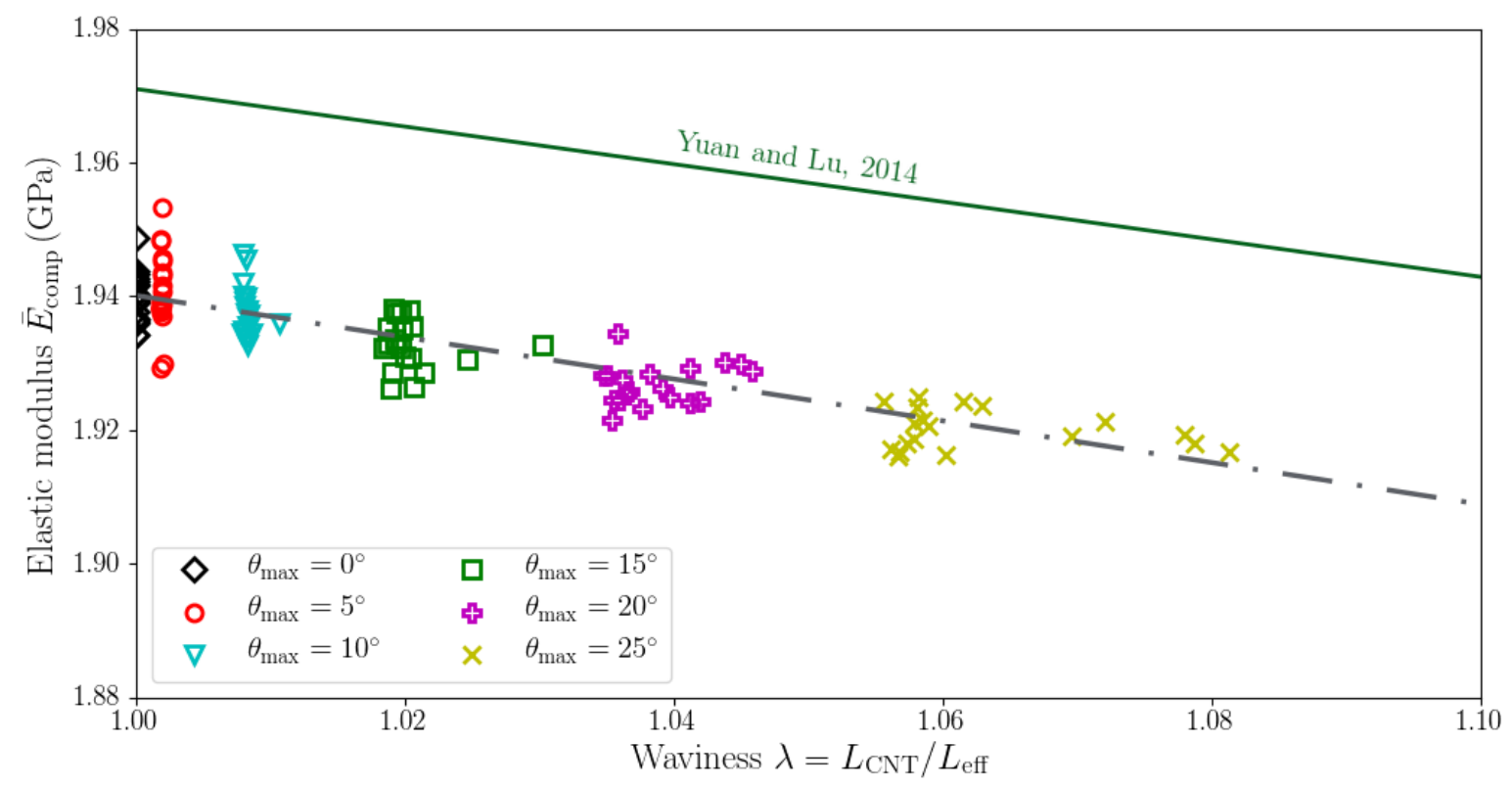

Fig. 7. Influence of the CNT waviness on the predicted homogenized Young's modulus.

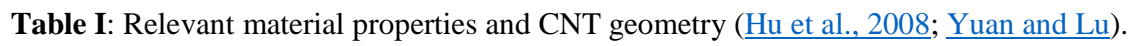

\begin{tabular}{c|c|c|c|c|c}
\hline \hline Material & $\begin{array}{c}\text { Elastic } \\
\text { modulus }\end{array}$ & $\begin{array}{c}\text { Poisson's } \\
\text { ratio }\end{array}$ & $\begin{array}{c}\text { Electrical } \\
\text { conductivity }\end{array}$ & Diameter & Length \\
\hline \hline SWNT & $1.0 \mathrm{TPa}$ & - & $10^{4} \mathrm{~S} / \mathrm{m}$ & $10 \mathrm{~nm}$ & $462 \mathrm{~nm}$ \\
\hline MWNT & $1.0 \mathrm{TPa}$ & - & $10^{4} \mathrm{~S} / \mathrm{m}$ & $50 \mathrm{~nm}$ & $5 \mu \mathrm{m}$ \\
\hline $\mathrm{PP}$ & $1.756 \mathrm{GPa}$ & 0.4 & - & - & - \\
\hline Epoxy & $1.0 \mathrm{GPa}$ & 0.35 & $10^{-6} \mathrm{~S} / \mathrm{m}$ & - & - \\
\hline \hline
\end{tabular}

\subsection{Electrical properties}

The proposed electrical model is now used to predict the electrical conductivity of a polymer nanocomposite with MWNTs, randomly dispersed in an epoxy matrix, as investigated by ( $\underline{\mathrm{Hu}} \mathrm{et}$ al. $)$. The MWNT dimensions considered by (ㅂu et al. $)$ are presented in Table I. We take a nanotube conductivity of $10^{4} \mathrm{~S} / \mathrm{m}$ and a (negligible) conductivity of $10^{-6} \mathrm{~S} / \mathrm{m}$ for the epoxy matrix. For 
the tunnelling junctions, we consider a relative permittivity of 3.98 (representative of Epoxy) and a work fraction for the MWNTs of $4.95 \mathrm{eV}$ (Shiraishi and Ata).

A preliminary study is conducted to explore the sensitivity of the homogenized conductivity to RVE size. This is performed for the case $V_{\mathrm{f}}=1 \%$; the RVE size is increased and the corresponding homogenized conductivity is presented in Fig. 8 for a converged number of 20 realizations. Predictions of the cross-terms of the conductivity matrix $\kappa_{i j}(i \neq j)$ are also presented, as they can be used to quantify the anisotropy of the RVE. The electrical conductivity $\bar{\kappa}_{\text {comp }}$ tends to a constant value with an increasing RVE size. Simultaneously, the cross-terms of the conductivity matrix of the RVE decrease. Based on the results in Fig. 8, we consider an RVE of size $L_{\mathrm{RVE}}=1.5 L_{\mathrm{CNT}}$ in the following.

For this choice of volume fraction and RVE size, a mesh convergence study resulted in a mesh with approximately 150,000 degrees of freedom. Meshes of similar densities are adopted when considering different volume fractions.

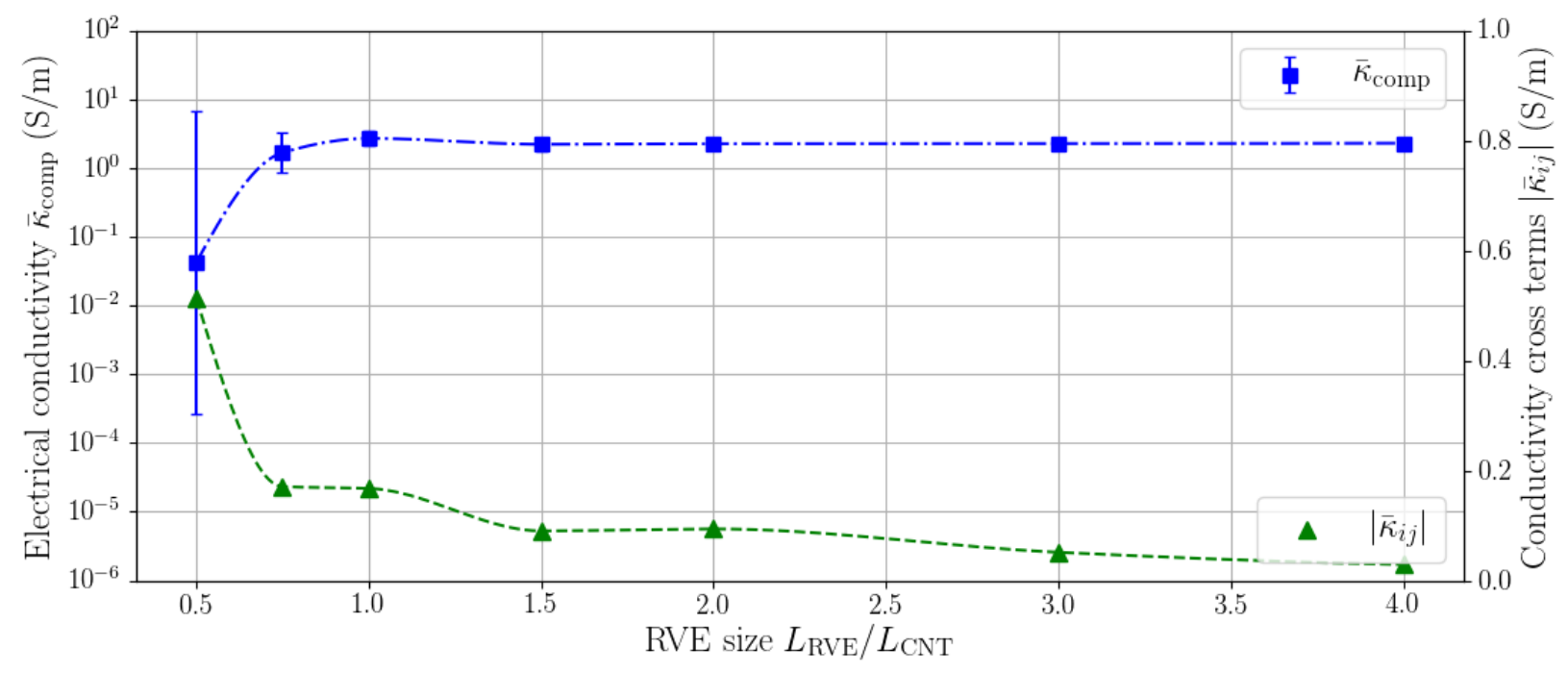

Fig. 8. Influence of the RVE size on the homogenized electrical conductivity.

The effect of the filler fraction on the electrical conductivity is now examined. Twenty realizations of the RVEs are simulated for each volume fraction and the average homogenized conductivity is plotted in Fig. 9. This Fig. 9 shows the typical response of a percolation problem (Sahimi, 2003), with three distinct regions. For low concentrations, the composite has a very small conductivity, as the matrix isolates the nanotubes. At the critical volume fraction $V_{\mathrm{f}_{\mathrm{c}}}$, the nanotubes 
become macroscopically connected (percolation occurs) and there is an abrupt increase in conductivity. After the percolation region, an increasing number of parallel conductive paths appear in the material as the volume fraction increases, and consequently the conductivity increases at a slower pace.

The volume fraction at percolation point is found to be at around $V_{\mathrm{f}_{\mathrm{c}}} \simeq 0.7 \%$, in agreement with the predictions obtained using the excluded volume theory for capped cylinders (Balberg et al.), $V_{\mathrm{f}_{\mathrm{c}}} \simeq 0.68 \%$ ). Furthermore, the standard deviation around the percolation point is very high. Since the CNTs begin to form an infinite cluster at the critical volume fraction, correspondingly the characteristic length of the problem tends to infinity (Stauffer and Aharony) and the anisotropy of the current distribution is also maximum. Since the CNTs begin to form an infinite cluster at the critical volume fraction, correspondingly the characteristic length of the problem tends to infinity (Stauffer and Aharony). This is in line with percolation theory, predicting that the correlation length and RVE size are proportional to $\left|V_{\mathrm{f}}-V_{\mathrm{f}_{\mathrm{c}}}\right|^{-\beta}$, with $\beta>0$ (Stauffer and Aharony). Consequently, it is expected that the critical RVE size tends to infinity and that the anisotropy of the current distribution is maximum around this point.

Fig. 9 includes experimental results from different authors, as reviewed by ( $\underline{\mathrm{Hu} \text { et al. }}$ ) These are in good agreement with the predicted response, especially at high volume fractions, confirming the effectiveness of the proposed framework to predict the bulk electrical conductivity of CNTpolymer composites. 


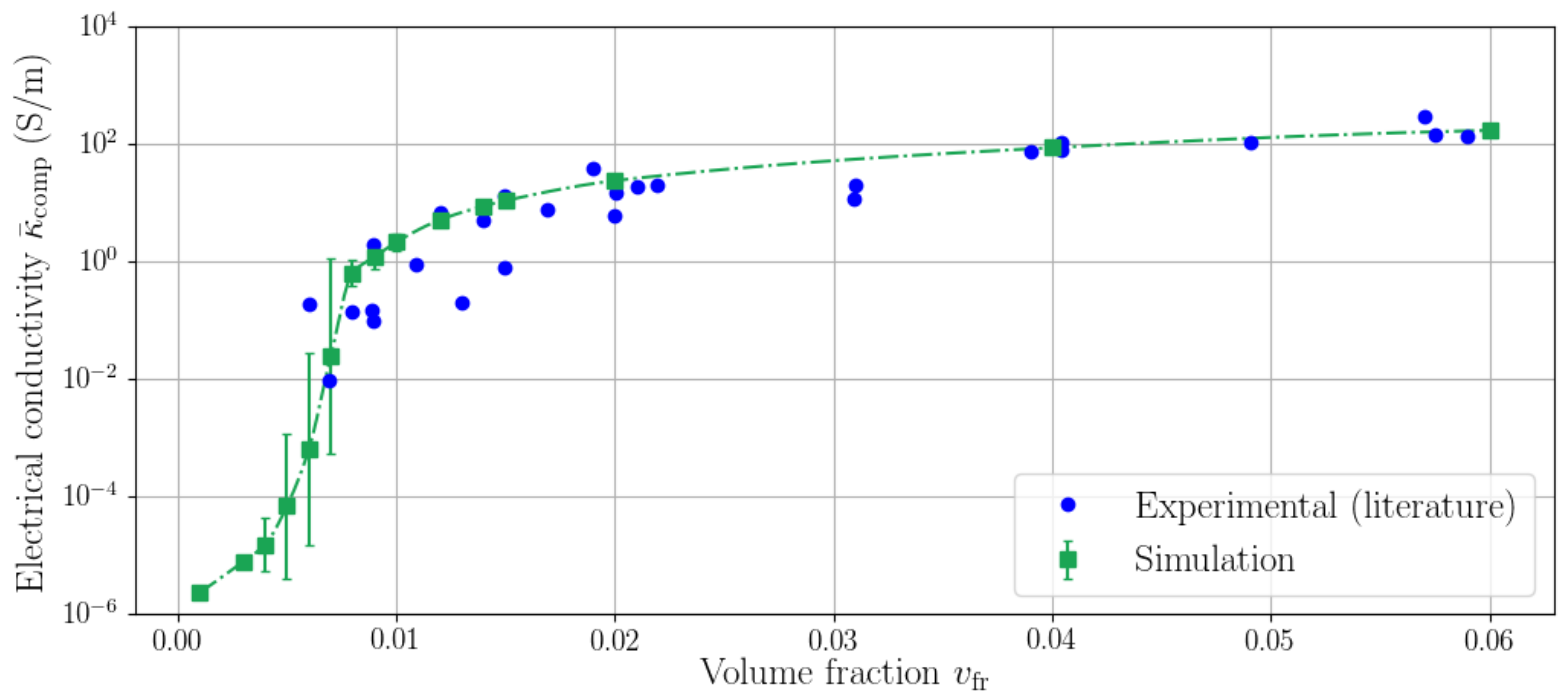

Fig. 9. Homogenized electrical conductivity as a function of the CNT volume fraction. The bars represent the standard deviation and the experimental data is reproduced from the review by ( $\underline{\mathrm{Hu} \text { et } \mathrm{al}}$.)

\subsection{Strain-sensing}

Finally, we explore the strain-sensing behaviour of a MWNT-epoxy composite material with a volume fraction of 1\%. Again, relevant material properties are given in Table I.

The predicted resistance change $\Delta R$, normalized by the initial resistance $R_{0}$, is plotted in Fig. 10 for an RVE with size $L_{\mathrm{RVE}}=3 \times L_{\mathrm{CNT}}=15 \mu \mathrm{m}$; this RVE size was determined in a convergence study. The figure includes three separate sets of predictions, corresponding to three different load cases: (i) uniaxial stress, (ii) uniaxial strain, (iii) volumetric strain. Measurements and simulations

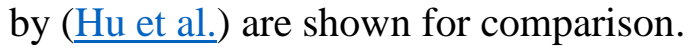




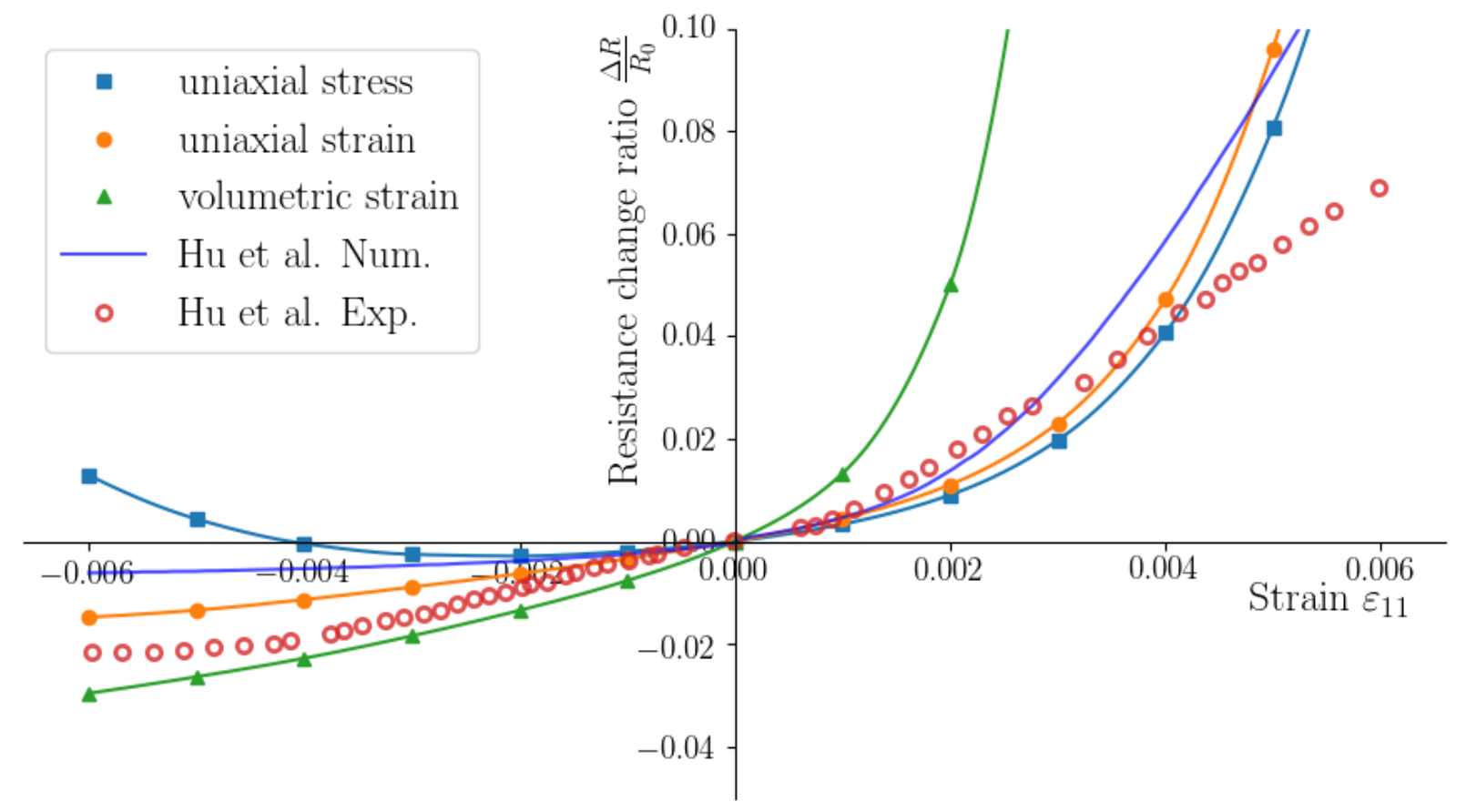

Fig. 10. Simulated piezoresistivity of a MWNT-epoxy composite with $1 \%$ volume fraction for uniaxial stress, uniaxial strain and volumetric strain, averaged over 20 realizations. Presented are also the experimental values by ( $\underline{\mathrm{Hu}}$ et al. $)$

The proposed models predict a significant variation of resistance with imposed strain, in all load cases examined. The predicted variations of resistance are of the same order of magnitude as those recorded in measurements; it should be noted that the boundary conditions in the experiments of ( $\underline{\mathrm{Hu} \text { et al. }}$ ) were not explicitly reported. The specimens used in ( $\underline{\mathrm{Hu} \text { et al. }}$ ) consist of relatively thin polymer films; consequently, we hypothesize that these were tested while adhered to a stiff substrate (to prevent buckling and wrinkling, especially in compression); consequently, their state of strain would be intermediate between those corresponding to uniaxial stress and uniaxial strain; this uncertainty prevents from ranking the accuracy of different modelling approaches. We also note that in real CNT-polymer composites the dispersion of CNTs is never perfectly uniform and the material presents agglomerates of CNTs, which are not accounted for in our analysis.

It should be noted that estimating the sensitivity of separation distance of tunnelling junctions to applied strain, $S_{k}^{i j}=\partial s_{k} / \partial \bar{\varepsilon}_{i j}$ is a well-known challenge, requiring a very dense meshing of the regions surrounding the junctions and an accurate description of the constitutive response of the materials at this length-scale $(\sim 1 \mathrm{~nm})$. While we addressed these challenges with local mesh convergence and parametric studies, multi-scale modelling techniques could be employed to overcome this problem; this is left as a topic for future studies. 
In tension, the RVE resistance increases with applied strain, as expected and reported by others (Gong and Zhu; Hu et al.; Pham et al., 2008); however, we observe that in compression, at small strains, the resistance decreases with increasing compressive strains; at larger compressive strains, the resistance may increase or decrease with the applied strain magnitude, depending on the boundary conditions. To further illustrate the reasons for this, in Fig. 11 we present, for the three different loading cases considered, the cumulative distributions of junction separations. Such distributions are provided for undeformed RVEs and for RVEs subject to tensile or compressive strains (in the direction of the applied electric field).

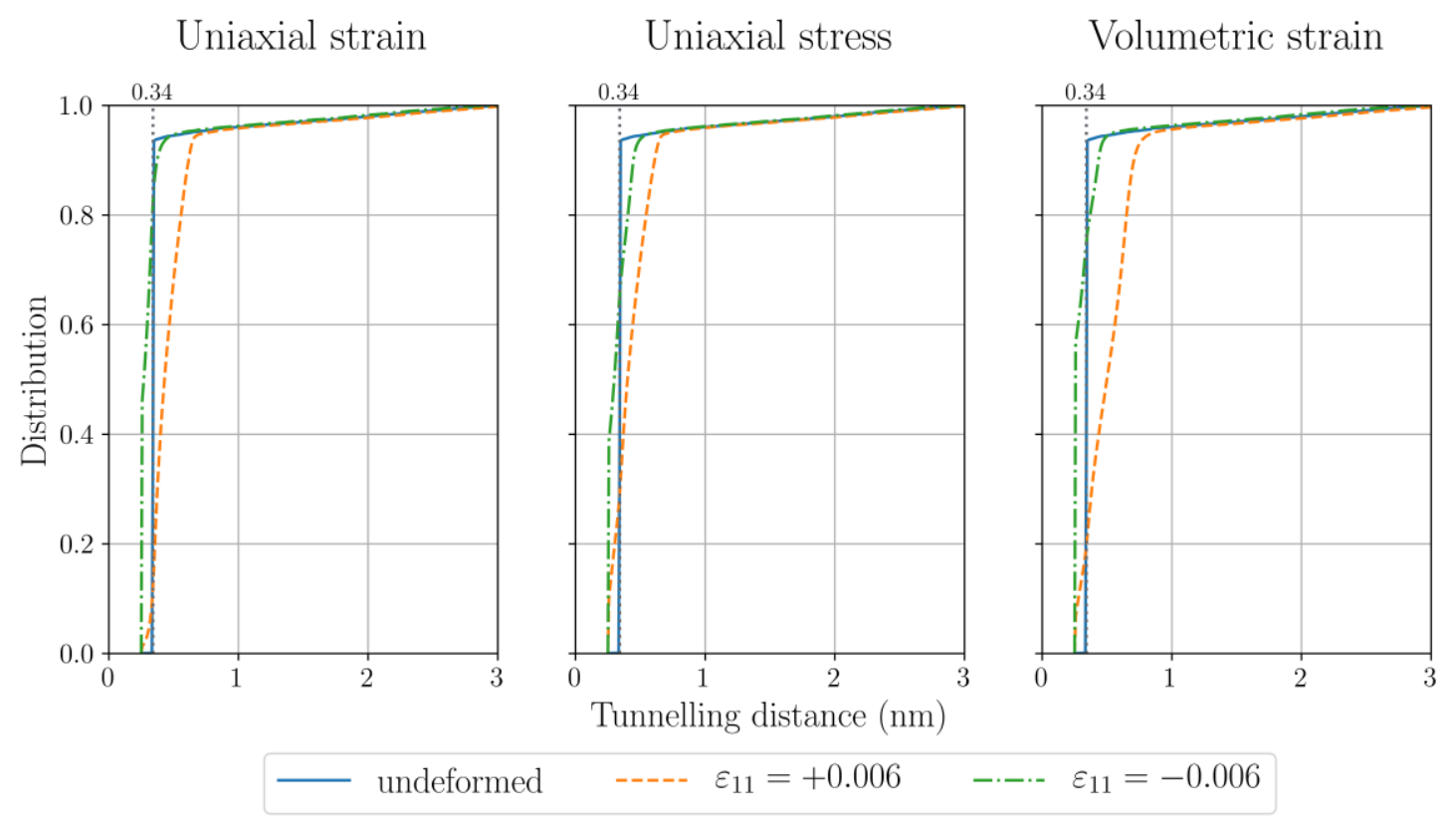

Fig. 11. Cumulative distributions of the junction separation $s$ for uniaxial strain, uniaxial stress and volumetric strain. The initial distribution is compared to those at positive or compressive strains

We observe that in compression, for all loading cases, the junction separation cannot decrease beyond the minimum value of $0.25 \mathrm{~nm}$, therefore limiting the strain-sensing capability of the composite. In both tension and compression, there is a subset of junctions with increased separation (contributing to a higher macroscopic resistance), and a complementary subset with decreasing separation (contributing to the opposite effect). The net change of resistance depends on the relative size of the populations in the two subsets, and this in turn is strongly affected by the load case. Fig. 11 shows that, in the case of uniaxial compressive stress, a relatively higher number of junctions is increasing their separation, corresponding (at large strains) to a net increase 
in macroscopic resistance. In contrast, for compressive volumetric or uniaxial strain, the number of junctions decreasing their separation is higher than those increasing it, which results in a decrease in macroscopic resistance.

In the case of imposed pure shear strain, the resistance change ratio is shown in Fig. 12(a) (averaged over 60 realizations). Two cases are presented, corresponding to the electric potential being applied perpendicularly or parallel to one the shear directions. The sensitivity of the resistance to shear strain is one order of magnitude smaller when compared to the cases in Fig. 10. Since the sensitivity to compressive strain is smaller than that to tensile strain, it is expected that the resistance change should be smaller but positive. Furthermore, since the distributions of CNTs is isotropic (random), the response is symmetric for positive and negative shear strains. This is confirmed by Fig. 12(b), showing that the distributions of separation distances under positive or negative shear strains are identical.

a)

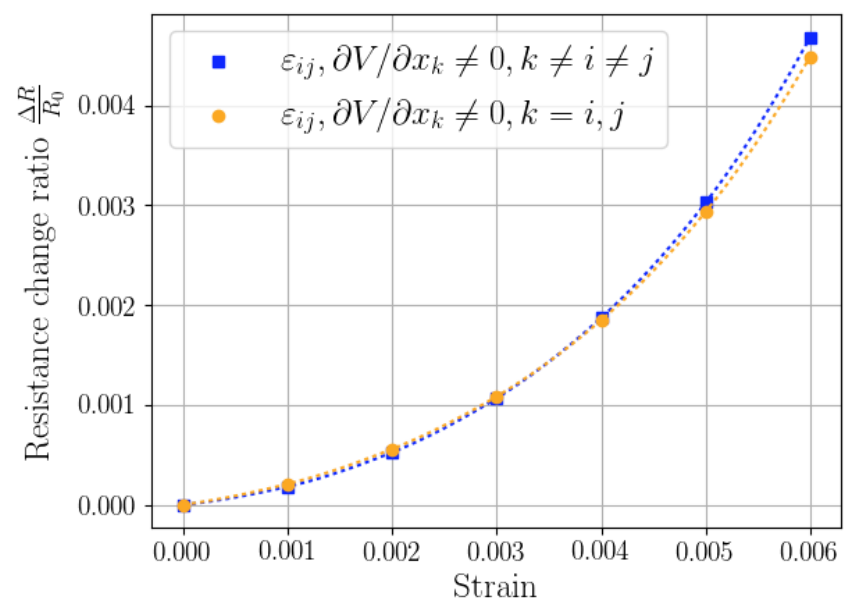

b)

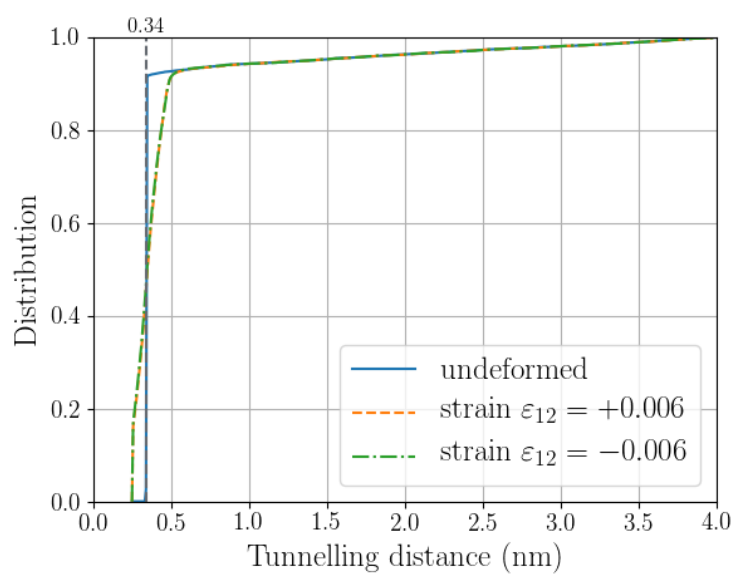

Fig. 12. Simulations of a) piezoresistivity of a MWNT-epoxy composite with $1 \%$ volume fraction for pure shear loading, averaged over 60 realizations and b) cumulative distributions of the junction separation $s$.

\section{Conclusions}

We presented FE calculations to predict the electro-mechanical response of CNT-polymer composites. Realistic RVEs were generated and Monte Carlo analyses of the electrical and mechanical response were performed. The numerical predictions were validated by comparison to published data and employed to explore the strain-sensing response of the composites in different load cases. The main conclusions of the study are as follows. 
- Modelling the CNTs as one-dimensional beams in a CNT-polymer composite with hollow cylindrical cross-section yields predictions of the elastic response in agreement with those obtained modelling the CNTs as three-dimensional solids.

- A novel finite element was developed, and then implemented in the commercial FE software Abaqus; this element captures tunnelling conductivity effects at the junctions between adjacent CNTs and does not employ fitting parameters to be calibrated against experimental results.

- The electrical conductivity percolation threshold of CNT-polymer composites can be accurately predicted by the proposed simulations; predictions are found in agreement with those of previously published analytical models. The sensitivity of conductivity to CNT volume fraction is also accurately captured.

- The strain-sensing response of the composites to pure shear deformation is negligible, in the ranges of strain and volume fractions investigated. The strain-sensing response in presence of positive or negative volumetric strains is strongly dependent on the loading case considered.

The physically-based models presented in this study may inspire and inform theoretical predictive models of the electro-mechanical response of CNT-polymer composites. With appropriate modifications, the models may also be employed to explore possible applications of CNT-polymer composites in damage detection. We leave these as topics for future investigations.

\section{Acknowledgments}

This project was funded by the European Union's Horizon 2020 research and innovation program, under the Marie Skłodowska-Curie Grant Agreement no. 642890 (http://thelinkproject.eu). 


\section{References}

Alamusi, Hu, N., Fukunaga, H., Atobe, S., Liu, Y.L., Li, J.H., 2011. Piezoresistive Strain Sensors Made from Carbon Nanotubes Based Polymer Nanocomposites. Sensors-Basel 11, 10691-10723. Balberg, I., Anderson, C.H., Alexander, S., Wagner, N., 1984. Excluded volume and its relation to the onset of percolation. Phys Rev B 30, 3933-3943.

Baughman, R.H., Zakhidov, A.A., de Heer, W.A., 2002. Carbon nanotubes - the route toward applications. Science 297, 787-792.

Bauhofer, W., Kovacs, J.Z., 2009. A review and analysis of electrical percolation in carbon nanotube polymer composites. Compos Sci Technol 69, 1486-1498.

Coleman, J.N., Khan, U., Blau, W.J., Gun'ko, Y.K., 2006. Small but strong: A review of the mechanical properties of carbon nanotube-polymer composites. Carbon 44, 1624-1652.

De Vivo, B., Lamberti, P., Spinelli, G., Tucci, V., Vertuccio, L., Vittoria, V., 2014. Simulation and experimental characterization of polymer/carbon nanotubes composites for strain sensor applications. Journal of Applied Physics 116, 054307.

Dharap, P., Li, Z.L., Nagarajaiah, S., Barrera, E.V., 2004. Nanotube film based on single-wall carbon nanotubes for strain sensing. Nanotechnology 15, 379-382.

Di Ventra, M., 2008. Electrical Transport in Nanoscale Systems. Cambridge University Press.

Ebbesen, T.W., Lezec, H.J., Hiura, H., Bennett, J.W., Ghaemi, H.F., Thio, T., 1996. Electrical conductivity of individual carbon nanotubes. Nature 382, 54-56.

Frogley, M.D., Zhao, Q., Wagner, H.D., 2002. Polarized resonance Raman spectroscopy of singlewall carbon nanotubes within a polymer under strain. Phys Rev B 65, 113413.

Fuhrer, M.S., Nygård, J., Shih, L., Forero, M., Yoon, Y.-G., Mazzoni, M.S.C., Choi, H.J., Ihm, J., Louie, S.G., Zettl, A., McEuen, P.L., 2000. Crossed Nanotube Junctions. Science 288, 494-497.

Girifalco, L.A., Hodak, M., Lee, R.S., 2000. Carbon nanotubes, buckyballs, ropes, and a universal graphitic potential. Phys Rev B 62, 13104-13110.

Gong, S., Zhu, Z.H., 2014. On the mechanism of piezoresistivity of carbon nanotube polymer composites. Polymer 55, 4136-4149.

Gong, S., Zhu, Z.H., Meguid, S.A., 2014. Carbon nanotube agglomeration effect on piezoresistivity of polymer nanocomposites. Polymer 55, 5488-5499.

Gonzalez, R.C., Woods, R.E., 2011. Digital Image Processing. Pearson Education.

Grabowski, K., Zbyrad, P., Uhl, T., Staszewski, W.J., Packo, P., 2017. Multiscale electromechanical modeling of carbon nanotube composites. Comp Mater Sci 135, 169-180.

Guo, X., Zhang, T., 2010. A study on the bending stiffness of single-walled carbon nanotubes and related issues. J Mech Phys Solids 58, 428-443.

Herasati, S., Zhang, L.C., 2014. A new method for characterizing and modeling the waviness and alignment of carbon nanotubes in composites. Compos Sci Technol 100, 136-142.

Hu, N., Karube, Y., Arai, M., Watanabe, T., Yan, C., Li, Y., Liu, Y.L., Fukunaga, H., 2010. Investigation on sensitivity of a polymer/carbon nanotube composite strain sensor. Carbon 48, 680-687.

Hu, N., Karube, Y., Yan, C., Masuda, Z., Fukunaga, H., 2008. Tunneling effect in a polymer/carbon nanotube nanocomposite strain sensor. Acta Mater 56, 2929-2936.

Huang, Y., Wu, J., Hwang, K.C., 2006. Thickness of graphene and single-wall carbon nanotubes. Phys Rev B 74.

Kilbride, B.E., Coleman, J.N., Fraysse, J., Fournet, P., Cadek, M., Drury, A., Hutzler, S., Roth, S., Blau, W.J., 2002. Experimental observation of scaling laws for alternating current and direct 
current conductivity in polymer-carbon nanotube composite thin films. Journal of Applied Physics 92, 4024-4030.

Kuronuma, Y., Takeda, T., Shindo, Y., Narita, F., Wei, Z.J., 2012. Electrical resistance-based strain sensing in carbon nanotube/polymer composites under tension: Analytical modeling and experiments. Compos Sci Technol 72, 1678-1682.

Mirzaeifar, R., Qin, Z., Buehler, M.J., 2015. Mesoscale mechanics of twisting carbon nanotube yarns. Nanoscale 7, 5435-5445.

Nigro, B., Grimaldi, C., 2014. Impact of tunneling anisotropy on the conductivity of nanorod dispersions. Phys Rev B 90.

Obitayo, W., Liu, T., 2012. A Review: Carbon Nanotube-Based Piezoresistive Strain Sensors. J Sensors, 15.

Odegard, G.M., Gates, T.S., Nicholson, L.M., Wise, K.E., 2002. Equivalent-continuum modeling of nano-structured materials. Compos Sci Technol 62, 1869-1880.

Oliva-Aviles, A.I., Aviles, F., Seidel, G.D., Sosa, V., 2013. On the contribution of carbon nanotube deformation to piezoresistivity of carbon nanotube/polymer composites. Composites Part BEngineering 47, 200-206.

Panozzo, F., Zappalorto, M., Quaresimin, M., 2017. Analytical model for the prediction of the piezoresistive behavior of CNT modified polymers. Composites Part B-Engineering 109, 53-63.

Park, J.M., Kim, D.S., Kim, S.J., Kim, P.G., Yoon, D.J., DeVries, K.L., 2007. Inherent sensing and interfacial evaluation of carbon nanofiber and nanotube/epoxy composites using electrical resistance measurement and micromechanical technique. Composites Part B-Engineering 38, 847861.

Park, M., Kim, H., Youngblood, J.P., 2008. Strain-dependent electrical resistance of multi-walled carbon nanotube/polymer composite films. Nanotechnology 19.

Pham, G.T., Park, Y.B., Liang, Z., Zhang, C., Wang, B., 2008. Processing and modeling of conductive thermoplastic/carbon nanotube films for strain sensing. Composites Part BEngineering 39, 209-216.

Rahman, R., Servati, P., 2012. Effects of inter-tube distance and alignment on tunnelling resistance and strain sensitivity of nanotube/polymer composite films. Nanotechnology 23, 055703.

Reddy, J.N., 2006. An Introduction To The Finite Element Method. McGraw-Hill.

Ren, X., Seidel, G.D., 2013. Computational micromechanics modeling of piezoresistivity in carbon nanotube-polymer nanocomposites. Composite Interfaces 20, 693-720.

Sahimi, M., 2003. Applications Of Percolation Theory. Taylor $1 \&$ Francis.

Sandler, J.K.W., Kirk, J.E., Kinloch, I.A., Shaffer, M.S.P., Windle, A.H., 2003. Ultra-low electrical percolation threshold in carbon-nanotube-epoxy composites. Polymer 44, 5893-5899.

Shiraishi, M., Ata, M., 2001. Work function of carbon nanotubes. Carbon 39, 1913-1917.

Simmons, J.G., 1963. Generalized Formula for the Electric Tunnel Effect between Similar Electrodes Separated by a Thin Insulating Film. Journal of Applied Physics 34, 1793.

Simmons, J.G., 1964. Generalized Thermal J- V Characteristic for the Electric Tunnel Effect. Journal of Applied Physics 35, 2655-2658.

Simulia, D.S., 2017a. Abaqus 2017 Documentation.

Simulia, D.S., 2017b. Abaqus User Subroutines Guide.

Spitalsky, Z., Tasis, D., Papagelis, K., Galiotis, C., 2010. Carbon nanotube-polymer composites: Chemistry, processing, mechanical and electrical properties. Prog Polym Sci 35, 357-401.

Stauffer, D., Aharony, A., 2003. Introduction To Percolation Theory. Taylor \& Francis. 
Suquet, P.M., 1987. Elements of Homogenization for Inelastic Solid Mechanics, Homogenization techniques for composite media. Springer-Verlag, pp. 193-278.

Taya, M., Kim, W.J., Ono, K., 1998. Piezoresistivity of a short fiber/elastomer matrix composite. Mech. Mater. 28, 53-59.

Thostenson, E.T., Chou, T.W., 2003. On the elastic properties of carbon nanotube-based composites: modelling and characterization. Journal of Physics D-Applied Physics 36, 573-582.

Tombler, T.W., Zhou, C., Alexseyev, L., Kong, J., Dai, H., Liu, L., Jayanthi, C.S., Tang, M., Wu, S.-Y., 2000. Reversible electromechanical characteristics of carbon nanotubes under local-probe manipulation. Nature 405, 769-772.

Treacy, M.M.J., Ebbesen, T.W., Gibson, J.M., 1996. Exceptionally high Young's modulus observed for individual carbon nanotubes. Nature 381, 678-680.

Wescott, J.T., Kung, P., Maiti, A., 2007. Conductivity of carbon nanotube polymer composites. Appl Phys Lett 90, 033116.

Wichmann, M.H.G., Buschhorn, S.T., Gehrmann, J., Schulte, K., 2009. Piezoresistive response of epoxy composites with carbon nanoparticles under tensile load. Phys Rev B 80, 8.

Wu, J., Hwang, K.C., Huang, Y., 2008. An atomistic-based finite-deformation shell theory for single-wall carbon nanotubes. J Mech Phys Solids 56, 279-292.

Xu, S., Rezvanian, O., Zikry, M.A., 2013. Electro-mechanical modeling of the piezoresistive response of carbon nanotube polymer composites. Smart Mater Struct 22, 12.

Yoon, Y.G., Mazzoni, M.S.C., Choi, H.J., Ihm, J., Louie, S.G., 2001. Structural deformation and intertube conductance of crossed carbon nanotube junctions. Phys. Rev. Lett. 86, 688-691.

Yuan, Z., Lu, Z., 2014. Numerical analysis of elastic-plastic properties of polymer composite reinforced by wavy and random CNTs. Comp Mater Sci 95, 610-619.

Zhang, W., Suhr, J., Koratkar, N., 2006. Carbon Nanotube/Polycarbonate Composites as Multifunctional Strain Sensors. J Nanosci Nanotechno 6, 960-964. 